\title{
Optimal control of vertically transmitted disease: an integrated approach
}

\author{
Samit Bhattacharyya ${ }^{\mathrm{a} *}$ and Suma Ghosh ${ }^{\mathrm{b}}$ \\ ${ }^{a}$ Mathematics and Statistics, University of Guelph, MacNaughton Building, 50 Stone Road, Guelph, \\ Ontario N1G 2W1, Canada; ${ }^{b}$ Mathematics and Statistics, York University, 4700 Keele Street, \\ Toronto, Ontario M3J 1P3, Canada
}

(Received 16 October 2009; final version received 3 July 2010)

\begin{abstract}
We study the dynamics of a disease under administration of a vaccine and antiviral drug, where the disease transmits directly from the parents to the offspring (vertical transmission) and also through contact with infective individuals (horizontal transmission). While vaccination to those susceptible reduces the horizontal transmission, administration of the antiviral drug to infected individuals lessens the chance of vertical transmission. Thus the vaccine and antiviral drug play different roles in controlling the disease, which has both vertical and horizontal transmission. We develop a 3D model with Susceptible-Infected-Recovered under vaccination to the susceptible and antiviral treatment to the infected and consider a control theoretic approach using the Pontryagin maximum principle to analyse the costeffectiveness of the control process. Our results demonstrate that a mixed intervention strategy of vaccination and antiviral drug in a proper ratio is the most effective way to control the disease. We show that cost-effectiveness of both intervention strategies intimately depends on disease-related parameters, such as force of infection, probability of being infected to offspring from infected mothers, loss of immunity or reinfection and also on cost of treatment.
\end{abstract}

Keywords: vertically transmitted disease; basic reproduction ratio; stability analysis; optimal control; Pontryagin maximum principle

AMS Subject Classification: 37N40; 97M60; 49J15; 92B05

\section{Introduction}

Infectious diseases in humans are caused by pathogenic microbial agents such as bacteria, virus, protozoa and fungi, which enter and infect a host individual. There are several infectious diseases where the infection transmits from one infected individual host to another susceptible through two distinct routes: horizontal transmission or direct contact and vertical transmission [8]. Horizontal transmission refers to the passage of the infection from one host individual to another, for example, by physical contact and by inhalation or ingestion of infective material. In contrast, vertical transmission refers to transmission of an infection from a mother to her offspring during the perinatal period, the period immediately before and after birth. A few good examples of such vertically transmitted diseases are HIV, hepatitis B or hepatitis C. These are few among all serious communicable diseases which burden worldwide these days. For example, approximately

\footnotetext{
*Corresponding author. Email: sbhattac@uoguelph.ca
} 
$30 \%$ of the world's population have serologic evidence of hepatitis B virus (HBV) infection. Of these, an estimated 350 million have chronic HBV infection, and at least 1 million chronically infected persons die each year from chronic liver disease, including cirrhosis and liver cancer. Even in a low endemic country such as the United States, hepatitis B causes more long-term sequelae and deaths than all other vaccine preventable childhood diseases, before the availability of vaccines to prevent this disease [16].

The literature dealing with the biological as well as mathematical theory on these diseases is quite extensive. The monograph of Bailey [1] and the survey article by Hethcote et al. [19] are devoted to the discussion on models of such diseases. Busenberg and Cooke [8] and Busenberg et al. [9] consider both continuous and discrete models of vertically communicable diseases. Some works on this can be found in the book by Thieme [31]. There are recent works [7,20,23,26,28,34] which focus on stability of such vertically transmitted disease dynamics. The concept of vertical transmission plays an important role in the cultural inheritance. This area has been studied by Cavalli-Sforza and Feldman [10], and their monograph describes the results obtained in this field.

A lot of effort on controlling such diseases with administration of antiviral treatment and vaccination has been taken up over the years [12,13,27,34]. For example, there are two types of hepatitis B vaccines (recombinant vaccine and hepatitis B immunoglobulin $\mathrm{G}$ ) and seven FDA-approved antiviral drugs available for hepatitis B, as well as interferon and ribavirin as vaccine and antiviral drug, respectively, for hepatitis C. Although a highly active anti-retroviral therapy is available for HIV [2,3], there is currently no publicly available vaccine or cure for HIV or AIDS. However, a vaccine that is a combination of two previously unsuccessful vaccines was reported, in September 2009, to have resulted in a $30 \%$ reduction in infections in a trial conducted in Thailand (http://news.bbc.co.uk/2/hi/ health/8272113.stm). There are a number of field studies and research going on to evaluate the effectiveness of several control measures and its administration [32]. Mathematical theories about this are almost as numerous [15]! But optimal management of controlling such diseases is comparatively less developed. In fact, an essential part in the control of such diseases is the consideration of economic viability of the process concerned. For instance, vaccination of infants in areas of high $\mathrm{HBV}$ prevalence is effective in reducing both infection and chronic carriage, but the cost-effectiveness of universal vaccination in low-risk areas such as the USA and Europe depends on a reduction in the cost of the vaccine [33]. HBV vaccination is most cost-effective when screening, and immunization of the newborn is combined with routine administration to all 10-year-old individuals [6]. There are works on optimal control on the ecological problems by Goh et al. [18] and Clark [11], etc. Especially, on the pest management problem, there are recent works by Bhattacharyya and Bhattacharya [4,5] and Ghosh and Bhattacharya [17], whereas its application on epidemiological problems is less developed.

In the present paper, we consider the dynamic model of a disease in a very generic sense, under administration of antiviral drug and vaccination to assess the clinical and economic impact of these two interventions. To study the basic reproduction ratio, stability of the solutions under realistic biological parameters and by the application of Pontryagin's maximum principle, we perform the optimal analysis of the control model considering the antiviral drug to the infective and vaccination to the susceptible as control parameters. The derivation of the model and its analysis are presented in Section 2. Section 3 considers the optimal analysis of the model and in Section 4, we consider some numerical experiments under special choice of parameter values and lastly we make certain conclusive remarks about the study. 


\section{Model and preliminaries}

We consider a three-compartment model consisting of Susceptible $(S)$, Infected $(I)$ and Recovered $(R)$ for a disease that propagates through contact between the infected and the susceptible individuals and also through the possibility of infected parents. We assume stable population with equal per capita birth and death rate $\mu$. There are many ways to model vertically transmitted disease $[8,23,28,34]$. For instance, Busenberg and Cooke [8] do not consider stable population. In fact, they assume susceptible and infected birth separately, and that population grows exponentially in the absence of infection. The horizontal transmission of disease propagation is denoted by the mass action term $k S I$, where $k$ represents the contact rate. For vertical transmission, we assume that a fraction $q_{1}$ of newborns from infected class are infected and it is denoted by the term $q_{1} \mu I,\left(q_{1}<1\right)$. Similarly, a fraction $q_{2}$ of newborns from recovered class are immune and it is denoted by $q_{2} \mu R,\left(q_{2}<1\right)$. Consequently, the birth flux into the susceptible class is given by $\mu-q_{1} \mu I-q_{1} \mu R$. As disease-induced death rate is not considered in the system, it is assumed that the total population is constant, i.e. $S(t)+I(t)+R(t)=1$. We consider that the infected class may undergo the antiviral treatment and switch to the recovered compartment proportional to the rate of drug uptake. Moreover, vaccination is administrated to the susceptible up to the younger age class and they move to recovered class. We also consider the loss of immunity and so there is an inflow from recovered class to susceptible class. A flow chart of this compartmental model is shown in Figure 1. These assumptions, however, lead to the following dynamic model:

$$
\begin{gathered}
\dot{S}=\mu-\mu S-k S I-q_{1} \mu I-q_{2} \mu R+\alpha R-m_{1} u_{1} S, \\
\dot{I}=k S I+q_{1} \mu I-\mu I-\gamma I-m_{2} u_{2} I, \\
\dot{R}=q_{2} \mu R-\mu R+\gamma I-\alpha R+m_{1} u_{1} S+m_{2} u_{2} I,
\end{gathered}
$$

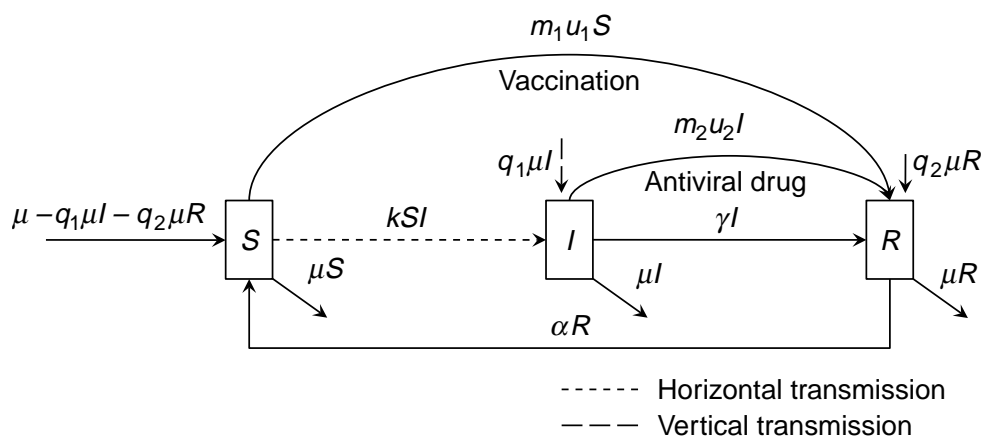

Figure 1. Flow diagram of disease dynamics under application of vaccine and antiviral drug. $S, I$ and $R$ denote three compartments of susceptible, infected and immune or recovered class, respectively. The term $k S I$ on the dotted line indicates the horizontal transmission from compartment $S$ to $I$, whereas $q_{1} \mu I$ on the dashed line denotes the vertical transmission from $I$ to $I$ by birth of offspring from an infected individual. Similarly, $q_{2} \mu R$ represents the proportions of immune newborn from recovered class. $\gamma I$ shows that individuals recover and move from compartment $I$ to $R$ and $\alpha R$ denotes a portion that moves from compartment $R$ to $S$ due to loss of immunity. Each compartment has their own death rate (in the slanted arrow). Curved arrows on top of the figure indicate proportions of susceptible and infected move to recovered class by vaccination and antiviral drug, respectively. 
where $k$ denotes the transmission rate, $\gamma$ indicates the rate of recovery of the infective and $\alpha$ denotes the rate of waning immunity or reinfection. The rate of vaccination and antiviral treatment is denoted by $u_{1}$ and $u_{2}$, whereas $m_{1}$ and $m_{2}$ indicate the efficacy of the respective control measures.

\subsection{Equilibria}

Models (1)-(3) possess mainly two equilibria:

- Disease-free equilibrium, $\bar{E}=(\bar{S}, 0, \bar{R})$, Where

$$
\begin{aligned}
\bar{S} & =\frac{\mu+\alpha-q_{2} \mu}{\mu+\alpha-q_{2} \mu+m_{1} u_{1}}, \\
\bar{R} & =\frac{m_{1} u_{1}}{\mu+\alpha-q_{2} \mu} \bar{S} .
\end{aligned}
$$

In the absence of vaccination, this reduces to the equilibrium $(1,0,0)$.

- Endemic equilibria, $E^{*}=\left(S^{*}, I^{*}, R^{*}\right)$, which has four different cases:

(i) No drug, no vaccine $\left(u_{1}=0, u_{2}=0\right), E_{1}^{*}=\left(S_{1}^{*}, I_{1}^{*}, R_{1}^{*}\right)$, where

$$
\begin{aligned}
& S_{1}^{*}=\frac{\mu+\gamma-q_{1} \mu}{k}, \\
& I_{1}^{*}=\frac{\left(\mu+\alpha-q_{2} \mu\right)\left[k-\left(\mu+\gamma-q_{1} \mu\right)\right]}{k\left(\gamma+\mu+\alpha-q_{2} \mu\right)}, \\
& R_{1}^{*}=\frac{\gamma I_{1}^{*}}{\mu+\alpha-q_{2} \mu} .
\end{aligned}
$$

(ii) With drug, no vaccine $\left(u_{1}=0, u_{2} \neq 0\right), E_{2}^{*}=\left(S_{2}^{*}, I_{2}^{*}, R_{2}^{*}\right)$, where

$$
\begin{aligned}
& S_{2}^{*}=\frac{\mu+\gamma+m_{2} u_{2}-q_{1} \mu}{k}, \\
& I_{2}^{*}=\frac{\left(\mu+\alpha-q_{2} \mu\right)\left[k-\left(\mu+\gamma+m_{2} u_{2}-q_{1} \mu\right)\right]}{k\left[\left(\gamma+m_{2} u_{2}\right)+\left(\mu+\alpha-q_{2} \mu\right)\right]}, \\
& R_{2}^{*}=\frac{\left(\gamma+m_{2} u\right) I_{2}^{*}}{\mu+\alpha-q_{2} \mu} .
\end{aligned}
$$

(iii) No drug, with vaccine $\left(u_{1} \neq 0, u_{2}=0\right), E_{3}^{*}=\left(S_{3}^{*}, I_{3}^{*}, R_{3}^{*}\right)$, here

$$
\begin{aligned}
& S_{3}^{*}=\frac{\mu+\gamma-q_{1} \mu}{k}, \\
& I_{3}^{*}=\frac{\left[k\left(\mu+\alpha-q_{2} \mu\right)-\left(\mu+\gamma-q_{1} \mu\right)\left(\mu+\alpha-q_{2} \mu+m_{1} u_{1}\right)\right]}{k\left[\gamma+\left(\mu+\alpha-q_{2} \mu\right)\right]}, \\
& R_{3}^{*}=\frac{m_{1} u_{1} S_{3}^{*}+\gamma I_{3}^{*}}{\mu+\alpha-q_{2} \mu} .
\end{aligned}
$$


(iv) With drug, with vaccine $\left(g_{1} \neq 0, g_{2} \neq 0\right), E_{4}^{*}=\left(S_{4}^{*}, I_{4}^{*}, R_{4}^{*}\right)$, where

$$
\begin{aligned}
& S_{4}^{*}=\frac{\mu+\gamma+m_{2} u_{2}-q_{1} \mu}{k}, \\
& I_{4}^{*}=\frac{\left[k\left(\mu+\alpha-q_{2} \mu\right)-\left(\mu+\gamma+m_{2} u_{2}-q_{1} \mu\right)\left(\mu+\alpha-q_{2} \mu+m_{1} u_{1}\right)\right]}{k\left[\left(\gamma+m_{2} u_{2}\right)+\left(\mu+\alpha-q_{2} \mu\right)\right]}, \\
& R_{4}^{*}=\frac{m_{1} u_{1} S_{4}^{*}+\left(\gamma+m_{2} u_{2}\right) I_{4}^{*}}{\mu+\alpha-q_{2} \mu} .
\end{aligned}
$$

Feasibility of $E_{4}^{*}$ requires

$$
k>\frac{\left(\mu+\gamma+m_{2} u_{2}-q_{1} \mu\right)\left(\mu+\alpha-q_{2} \mu+m_{1} u_{1}\right)}{\left(\mu+\alpha-q_{2} \mu\right)} .
$$

Feasibility of other equilibria $E_{i}^{*}, i=1,2,3$, may be obtained from (18) by assuming $u_{1}$ and/or $u_{2}=0$.

\subsubsection{Reproduction ratio}

The basic reproduction ratio $R_{0}$ for the disease transmission in the above model is given by

$$
R_{0}\left(k, q_{1}\right)=\frac{k}{\mu+\gamma-q_{1} \mu}
$$

which upon Taylor expansion gives

$$
R_{0}\left(k, q_{1}\right)=\frac{k}{\mu+\gamma}\left[1+v+v^{2}+\ldots\right],=R_{00}\left[1+v+v^{2}+\ldots\right]
$$

where

$$
R_{00}=\frac{k}{\mu+\gamma} \quad v=\frac{q_{1} \mu}{\mu+\gamma}
$$

An interpretation of $R_{0}$ is given as follows: over the mean infectious period $1 /(\mu+\gamma)$, a single infection produces $k .1 /(\mu+\gamma)$ infected individuals through direct contact or horizontal transmission. This is given by the first term of $R_{0}$. Now each of these new infected gives birth to $\mu /(\mu+\gamma)$ offspring during the mean infectious period and out of which $\mu /(\mu+\gamma) \cdot q_{1}$ are infected offspring. So, the total newly infected offspring are $k .1 /(\mu+\gamma) \cdot \mu /(\mu+\gamma) \cdot q_{1}$ This is exactly the second term of the expression in Equation (20). Similarly the third and higher ordered terms represent the contribution through vertical transmission in the third generation and so on from the same group of infected individuals. 
We also define the control reproduction ratio $R_{\mathrm{c}}$ by

$$
R_{\mathrm{c}}=\frac{k\left(\mu+\alpha-q_{2} \mu\right)}{\left(\mu+\gamma-q_{1} \mu+m_{2} u_{2}\right)\left(\mu+\alpha-q_{2} \mu+m_{1} u_{1}\right)} .
$$

Note that administration of either vaccine $u_{1}$ or antiviral drug $u_{2}$ reduces the value of $R_{\mathrm{c}}$.

Remark 1. It may be observed from relation (19) that the equilibrium $E_{1}^{*}$ is positive, if $R_{0}>1$ holds, i.e. if $k+q_{1} \mu>\mu+\gamma$. This with (23) clears the fact that both horizontal and vertical transmission play a substantial role in persistence of disease and it persists only if both horizontal and vertical transmission should be sufficiently higher than a certain threshold value dependent on birth and death of individuals. Relation (23) quantifies their separate contribution in disease transmission. We should also note that $R_{0} \rightarrow 1$ implies $E_{1}^{*} \rightarrow(1,0,0)$, the disease-free equilibrium and at $R_{0}=1$, we get $E_{1}^{*}=(1,0,0)$.

Remark 2. It is clear from (20) that endemic equilibrium $E_{i}^{*}$ is stable if respective control reproduction ratio $R_{\mathrm{c}}$ is greater than 1 . We should note from (20) that application of vaccine and antiviral drug both reduce the value of $R_{\mathrm{c}}$, and simultaneous effects of both intervention strategies on $R_{\mathrm{c}}$ are not simply the addition of two independent effects, rather they multiply each other to enhance the sum of independent effects in population level. Here lies the advantage for administration of integrated control. We show in numerical simulation how $R_{\mathrm{c}}$ is regulated by different rates of $u_{1}$ and $u_{2}$. However, the threshold values for rate of vaccine $u_{1}$ and antiviral drug $u_{2}$ to reduce $R_{\mathrm{c}}$ to less than 1 are given by

$$
\begin{gathered}
u_{1}^{*}=\frac{\left(\mu+\alpha-q_{2} \mu\right)\left\{k-\left(\mu+\gamma-q_{1} \mu\right)\right\}}{m_{1}\left(\mu+\gamma-q_{1} \mu\right)}, \\
u_{2}^{*}=\frac{1}{m_{2}\left\{k-\left(\mu+\gamma-q_{1} \mu\right)\right\}},
\end{gathered}
$$

respectively. Thus, effectiveness of vaccination naturally depends on the rate of waning immunity $\alpha$, proportion of immune newborns $q_{2}$, etc.

\subsection{Stability analysis}

In this section, we will discuss the stability of different equilibria. The variational matrix of the linearized system is given by

$$
\mathbf{J}=\left(\begin{array}{ccc}
-k I-\mu-m_{1} u_{1} & -k S-q_{1} \mu & a-q_{2} \mu \\
k I & k S-\mu-\gamma+q_{1} \mu-m_{2} u_{2} & 0 \\
m_{1} u_{1} & \gamma+m_{2} u_{2} & q_{2} \mu-a-\mu
\end{array}\right)
$$

The characteristic equation for disease-free equilibrium $\bar{E}$ is

$$
\left(\lambda-\lambda_{1}\right)\left\{\left(\lambda+\mu+m_{1} u_{1}\right)\left(\lambda+\mu+\alpha-q_{2} \mu\right)-m_{1} u_{1}\left(\alpha-q_{2} \mu\right)\right\},
$$


where $\lambda_{1}=k \bar{S}-\mu-\gamma+q_{1} \mu-m_{2} u_{2}$. This gives one root as, which is negative if

$$
R_{\mathrm{c}}<1 \text {. }
$$

The other two roots are given by the polynomial $\lambda^{2}+\left\{\left(\mu+m_{1} u_{1}\right)+\left(\mu+\alpha-q_{2} \mu\right)\right\} \lambda+$ $\left(\mu+m_{1} u_{1}\right)\left(\mu+\alpha-q_{2} \mu\right)-m_{1}\left(\alpha-q_{2} \mu\right)=0$ and they have negative real parts.

The characteristic equation for interior equilibrium $E_{i}^{*}(i=2, \ldots, 4)$ is given by

$$
\lambda^{3}+a_{2} \lambda^{2}+a_{1} \lambda+a_{0}=0
$$

where

$$
\begin{gathered}
a_{2}=\mu+m_{1} u_{1}+\alpha+\mu-q_{2} \mu+k I_{i}^{*}, \\
a_{1}=\left(\mu+k I_{i}^{*}+m_{1} u_{1}\right)\left(\mu+\alpha-q_{2} \mu\right)+\left(k S_{i}^{*}+q_{1} \mu\right) k I_{i}^{*}+m_{1} u_{1}\left(q_{2} \mu-\alpha\right), \\
a_{0}=\left(\mu+\alpha-q_{2} \mu\right)+\left(k S_{i}^{*}+q_{1} \mu\right) k_{i}^{*}+\left(q_{2} \mu-\alpha\right) k I_{i}^{*}\left(\gamma+m_{2} u_{2}\right), \quad i=1,2,3,4 .
\end{gathered}
$$

It is seen that $a_{j}>0$ for all $j=0,1,2$ and $a_{2} a_{1}-a_{0}>0$, if

$$
R_{\mathrm{c}}>1
$$

and hence by Routh-Hurwitz criterion, $E_{i}^{*}$ is stable (for all $i=2, \ldots, 4$ ).

Remark 3. It is interesting to note that $a_{0}=0$, whenever $R_{\mathrm{c}}=1$. In fact, the system converges to disease-free equilibrium $\bar{E}$ when $R_{\mathrm{c}}<1$ and it switches to endemic equilibrium $E_{i}^{*}$ through saddle-node bifurcation at $R_{\mathrm{c}}=1$. Endemic equilibrium $E_{i}^{*}$ exists and is stable when $R_{\mathrm{c}}>1$.

Remark 4. Stability of $E_{1}^{*}$ is assured similarly, if $R_{0}>1$.

\section{Optimization of the control policy}

So far, we have seen that under some suitable threshold limits of different parameters, the 3D SIR model system is locally asymptotically stable around the endemic equilibrium $E_{4}^{*}$. But during the process of applying control measures such as vaccination or antiviral treatment, there is an obvious question of incurring some cost and allied benefit in the whole process. Thus the objective is to quantify the units to express the net profit during the given time of treatment. In other words, this is to construct an economic model out of the given dynamic model of control. In this case the problem reduces to an optimal control problem. Our task is then to formulate an optimal policy when the control measures in the system are already defined in a mathematical form and finally to find out the restrictions on the economic parameters of the model. We rewrite the model as

$$
\begin{aligned}
& \dot{S}=\mu-\mu S-k S I-q_{1} \mu I-q_{2} \mu R+\alpha R-U_{1}, \\
& \dot{I}=k S I-\mu I+q_{1} \mu I-\gamma I-U_{2}, \\
& \dot{R}=q_{2} \mu R-\mu R+\gamma I-\alpha R+U_{3},
\end{aligned}
$$

where $U_{i}$ s are the total effects (gain/loss) to the growth equations of susceptible, infected and recovered, respectively, due to simultaneous use of vaccine and antiviral drug. We still assume that $u_{1}$ and $u_{2}$ should satisfy all the inequalities to make the system stable. 


\subsection{Formulation of the objective function}

The formulation of the objective function $\pi=\pi(S, I, R)$, which is to be maximized over the set of control parameters, is explained as follows:

Let $c_{\mathrm{V}}$ be the cost per unit vaccine and $c_{\mathrm{M}}$ be the cost per unit antiviral drug to be applied. $p$ denotes the projected price of the individual candidate in the system in terms of their social value.

D1: Profit due to implementation of vaccination and antiviral treatment $=p R U_{3}$.

D2: Net expenditure for application of vaccine of amount $m_{1} u_{1}$ over the loss incurred due to efflux of the susceptible $=c_{\mathrm{v}} m_{1} u_{1}-p S U_{1}=\left(c_{\mathrm{V}} / S-p S\right) U_{1}$.

D3: Net expenditure for application of antiviral treatment of amount $m_{2} u_{2}$ over the loss incurred due to efflux of infected $=c_{\mathrm{M}} m_{2} u_{2}-p I U_{2}=\left(c_{\mathrm{M}} / I-p I\right) U_{2}$.

Hence, the objective function for net benefit out of the control process is given by

$$
\begin{aligned}
\pi & =p R U_{3}-\left(\frac{c_{\mathrm{v}}}{S}-p S\right) U_{1}-\left(\frac{c_{\mathrm{M}}}{I}-p I\right) U_{2}, \\
& =\left(p S-\frac{c_{\mathrm{v}}}{S}\right) U_{1}+\left(p I-\frac{c_{\mathrm{M}}}{I}\right) U_{2}+p R U_{3} .
\end{aligned}
$$

After the formulation of the objective function, our next task is to find out the functions $U_{i}(t)$ which drive the dynamical system (28)-(30) from its initial state to a steady-state optimal solution $\left(S^{* *}, I^{* *}, R^{* *}\right)$ so as to maximize the integral

$$
\mathcal{J}\left(U_{1}, U_{2}, U_{3}\right)=\int_{0}^{T} \pi\left(S, I, R, U_{i}, t\right) \mathrm{d} t,
$$

over

$$
\Gamma=\left\{\left(U_{1}, U_{2}, U_{3}\right) \mid 0 \leq U_{i}(t) \leq m_{i} u_{i}^{*}, \quad i=1,2 ; 0 \leq U_{3}(t) \leq m_{1} u_{1}^{*}+m_{2} u_{2}^{*}\right\},
$$

where $T$ is the total time of applying both measures as shown in system (28)-(30). We may point out that this steady state is a singular extremal in this case, because the control variables $U_{i}$ appear linearly in the system of Equations (28)-(30) and objective functional (31). Applying Pontryagin's maximum principle on the constructed Hamiltonian $H$, we obtain the optimal steady-state solution $\left(S^{* *}, I^{* *}, R^{* *}\right)$ and corresponding control vector $U_{i}^{*}$. Moreover, the main objective in this optimal control problem is to maximize $\mathcal{J}$ (which is the same as minimizing the cost of vaccine and treatment). Thus, the generalized Legendre condition requires that along the singular solution, the matrix $\left(a_{i j}\right)$ with

$$
a_{i j}=\frac{\partial}{\partial u_{i}}\left(D^{2}\left(\frac{\partial H}{\partial u_{j}}\right)\right) \quad(i, j=1,2,3,4)
$$

must be negative semi-definite at $\left(S^{* *}, I^{* *}, R^{* *}\right)$. This condition of semi-definiteness of the above matrix imposes some restrictions on the economic parameters of the model, which means that the cost and the allied benefit or gain in the process must have some necessary limitations to get the maximum benefit or minimum cost of the whole process.

In this connection, we may point out that necessary conditions are more useful than the sufficient conditions in the application of optimal control theory. This is because it is extremely difficult to apply sufficient conditions in a real-world problem. There are, in fact, several sets of necessary conditions in optimal control theory in ecological problems, which in turn reflects the complexity in ecological systems. For example, a set of 
necessary conditions in this regard may be found in Goh et al.'s book [18]. The most useful set of necessary conditions for singular control consists of the generalized Legendre conditions. By applying this condition we have the following theorem.

THeOREM 3.1. Consider the objective functional $\mathcal{J}$ given by Equation (31) with $\left(U_{1}, U_{2}, U_{3}\right) \in \Gamma$ constraint to the state system (28)-(30). Then there exists $\left(U_{1}^{*}, U_{2}^{*}, U_{3}^{*}\right) \in \Gamma$ such that $\mathcal{J}\left(U_{1}^{*}, U_{2}^{*}, U_{3}^{*}\right)=\max \left\{\mathcal{J}\left(U_{1}, U_{2}, U_{3}\right) \mid\left(U_{1}, U_{2}, U_{3}\right) \in \Gamma\right\}$, if the following conditions

$$
\begin{gathered}
p<\frac{2 S^{* * 3}\left(\mu+k I^{* *}\right)-c_{\mathrm{v}}\left(\mu-q_{1} \mu\right) I^{* *}}{S^{* * 2}\left(\mu-q_{1} \mu\right) I^{* *}}, \\
I^{* *}>\max \left[\frac{\left(p S^{* *}-\frac{c_{\mathrm{v}}}{S^{* *}}\right)\left(k S^{* *}+q_{1} \mu\right)}{2 p\left(k S^{* *}+\left(q_{1} \mu-\mu-\gamma\right)\right)}, \frac{\left(p S^{* *}-\frac{c_{\mathrm{v}}}{S^{* *}}\right)\left(\alpha-q_{2} \mu\right)}{p \gamma}\right]
\end{gathered}
$$

are satisfied.

ProOF. We transform the integral occurring in $I$ by choosing

$$
f_{1} \equiv f_{1}(S), \quad f_{2} \equiv f_{2}(I), \quad f_{3} \equiv f_{3}(R),
$$

such that $f_{1}(S(0))=0, f_{2}(I(0))=0, f_{3}(R(0))=0$,

$$
\begin{aligned}
& \frac{d}{\mathrm{~d} t}\left[f_{1}(S)\right]=\left(p S-\frac{c_{\mathrm{v}}}{S}\right) \dot{S}, \\
& \frac{d}{\mathrm{~d} t}\left[f_{2}(I)\right]=\left(p I-\frac{c_{\mathrm{M}}}{I}\right) \dot{I}, \\
& \frac{d}{\mathrm{~d} t}\left[f_{3}(R)\right]=p R \dot{R} .
\end{aligned}
$$

Then

$$
\begin{aligned}
\mathcal{J}= & \int_{0}^{T} \pi(S, I, R, U) \mathrm{d} t \\
= & \int_{0}^{T}\left[\left(p S-\frac{c_{\mathrm{V}}}{S}\right)\left\{\mu-\mu S-k S I-q_{1} \mu I-q_{2} \mu R+\alpha R-\dot{S}\right\}\right. \\
& \left.\quad+\left(p I-\frac{c_{\mathrm{M}}}{I}\right)\left\{k S I-\mu I+q_{1} \mu I-\gamma I-\dot{I}\right\}-p R\left\{q_{2} \mu R-\mu R+\gamma I-\alpha R-\dot{R}\right\}\right] \mathrm{d} t, \\
= & \int_{0}^{T} W(S, I, R) \mathrm{d} t-\int_{0}^{T}\left[\frac{d}{\mathrm{~d} t}\left(f_{1}(S)\right)+\frac{d}{\mathrm{~d} t}\left(f_{2}(I)\right)-\frac{d}{\mathrm{~d} t}\left(f_{3}(R)\right)\right] \mathrm{d} t, \\
= & \int_{0}^{T} W(S, I, R) \mathrm{d} t-\left[f_{1}(S(T))+f_{2}(I(T))-f_{3}(R(T))\right],
\end{aligned}
$$

where

$$
W(S, I, P)=A \phi+B \psi-C .
$$


$A=\left(p S-\frac{c_{V}}{S}\right), \quad B=\left(p I-\frac{c_{M}}{I}\right), \quad C=p R\left\{q_{2} \mu R-\mu R+\gamma I-\alpha R\right\}, \quad \phi=\mu-\mu S-k S I$ $-q_{1} \mu I-q_{2} \mu R+\alpha R$ and $\psi=k S I-\mu I+q_{1} \mu I-\gamma I$.

Now we consider the Hamiltonian

$$
H=W(S, I, R, t)+\sum_{i=1}^{2} \lambda_{i}\left(G_{i}-U_{i}\right)+\lambda_{3}\left(G_{3}+U_{3}\right)
$$

where $G_{1}=\mu-\mu S-k S I-q_{1} \mu I-q_{2} \mu R+\alpha R, G_{2}=k S I-\mu I+q_{1} \mu I-\gamma I, G_{3}=$ $q_{2} \mu R-\mu R+\gamma I-\alpha R$ and $\lambda_{i}$ are co-state variables to be determined suitably. For steadystate solution, we have

$$
G_{i}-U_{i}=0, \quad i=1,2,
$$

and

$$
G_{3}+U_{3}=0
$$

If we suppose that there exists $\left(U_{1}^{*}, U_{2}^{*}, U_{3}^{*}\right)$ for which $\left(S^{* *}, I^{* *}, R^{* *}\right)$ gives a steady-state optimal solution of (28)-(30), then from Pontryagin's maximum principle, it follows that at this steady state, we have

$$
\begin{aligned}
& \dot{\lambda}_{1}=-\frac{\partial H}{\partial S}=-\frac{\partial W}{\partial S}-\sum_{i=1}^{3} \lambda_{i} \frac{\partial G_{i}}{\partial S}, \\
& \dot{\lambda}_{2}=-\frac{\partial H}{\partial I}=-\frac{\partial W}{\partial I}-\sum_{i=1}^{3} \lambda_{i} \frac{\partial G_{i}}{\partial I}, \\
& \dot{\lambda}_{3}=-\frac{\partial H}{\partial P}=-\frac{\partial W}{\partial R}-\sum_{i=1}^{3} \lambda_{i} \frac{\partial G_{i}}{\partial R},
\end{aligned}
$$

and

$$
\frac{\partial H}{\partial U_{i}}=0
$$

at $\left(U_{1}^{*}, U_{2}^{*}, U_{3}^{*}\right)$. This implies that $\lambda_{i}=0$. The control vector $U$ appears linearly in the Hamiltonian. Therefore, $\left(U_{1}^{*}, U_{2}^{*}, U_{3}^{*}\right)$ is a singular control variable. Now, the necessary conditions of optimality further ensure that at this singular control

$$
\begin{gathered}
D\left(\frac{\partial H}{\partial U_{i}}\right)=0 \\
\frac{\partial}{\partial U_{j}}\left(D\left(\frac{\partial H}{\partial U_{i}}\right)\right)=0,
\end{gathered}
$$

which, in turn, shows that $\dot{\lambda}_{i}=0$. This further implies through (39)-(41) that

$$
\frac{\partial W}{\partial S}=0, \frac{\partial W}{\partial I}=0, \frac{\partial W}{\partial R}=0
$$


which by (35) reduces to

$$
\begin{gathered}
p\left(\mu-2 \mu S-2 k S I-q_{1} \mu I+F R\right)+\frac{c_{\mathrm{v}}}{S^{2}}\left(\mu-q_{1} \mu I+F R\right)+k\left(p I^{2}-c_{\mathrm{M}}\right)=0, \\
\left(k s+q_{1} \mu\right)\left(p S-\frac{c_{\mathrm{v}}}{S}\right)+2 p\left(k S I+\left(q_{1} \mu-\mu-\gamma\right) I\right)-p \gamma R=0, \\
\left(p S-\frac{c_{\mathrm{v}}}{S}\right) F-p \gamma I+2 p\left(\alpha+\mu-q_{2} \mu\right) R=0,
\end{gathered}
$$

where $F=\alpha-q_{2} \mu$. Using Equations (45)-(47), we get the optimal equilibrium or nontrivial bionomic equilibrium $\left(S^{* *}, I^{* *}, R^{* *}\right)$ under the conditions:

$$
\begin{aligned}
& p<\frac{2 S^{* * 3}\left(\mu+k I^{* *}\right)-c_{\mathrm{v}}\left(\mu-q_{1} \mu\right) I^{* *}}{S^{* * 2}\left(\mu-q_{1} \mu\right) I^{* *}}, \\
& I^{* *}>\max \left\{\frac{\left(p S^{* *}-\frac{c_{\mathrm{v}}}{S^{* *}}\right)\left(k S^{* *}+q_{1} \mu\right)}{2 p\left(k S^{* *}+\left(q_{1} \mu-\mu-\gamma\right)\right)}, \frac{\left(p S^{* *}-\frac{c_{\mathrm{v}}}{S^{* *}}\right)\left(\alpha-q_{2} \mu\right)}{p \gamma}\right\} .
\end{aligned}
$$

These are exactly the conditions (33) and (34). Using the values of $S^{* *}, I^{* *}, R^{* *}$ on model Equations (28) and (29), we find out the values of singular control $\left(U_{1}^{*}, U_{2}^{*}, U_{3}^{*}\right)$, which give the estimates on the rates of vaccination $u_{1}$ and antiviral treatment $u_{2}$ for optimum control of the disease in the population. These inequalities (33) and (34), however, define the constraints on disease-related parameters as well as economic parameters such as cost of vaccine and medicines and also proportion of population to be vaccinated or to be given antiviral treatment.

We can also find out the necessary condition for optimality by showing that the matrix $\mathbf{L}=\left(a_{i j}\right)$ is negative semi-definite at $\left(S^{* *}, I^{* *}, R^{* *}\right)$, where

$$
a_{i j}=\frac{\partial}{\partial U_{i}}\left(D^{2}\left(\frac{\partial H}{\partial U_{j}}\right)\right)=-\frac{\partial^{2} W}{\partial S \partial I}, \quad(i, j=1, \ldots, 3), \text { etc. }
$$

Differentiating (38) twice with respect to $S, I, R$, we get

$$
\begin{aligned}
& a_{11}=-2\left[p(\mu+k I)+\frac{c_{\mathrm{v}}}{S^{3}}(\mu-q \mu I+F R)\right], \\
& a_{22}=2 p\left(k S+q_{1} \mu-\mu-\gamma\right), \\
& a_{33}=2 p\left(\alpha+\mu-q_{2} \mu\right) \\
& a_{12}=a_{21}=-2 k p S-\left(p+\frac{c_{\mathrm{v}}}{S^{2}}\right)+2 k p I, \\
& a_{13}=a_{31}=\left(p+\frac{c_{\mathrm{v}}}{S^{2}}\right) F \\
& a_{23}=a_{32}=-p \gamma .
\end{aligned}
$$

Thus, negative semi-definiteness of matrix $\mathbf{L}$ may be obtained by making $a_{11}<0$, $a_{11} a_{22}-a_{12}^{2}>0$ and $\operatorname{det}\left(a_{i j}\right)<0$ using the above expressions.

Remark 1. We may note that inequalities (33) and (34) and inequalities for negative semidefiniteness of matrix $\mathbf{L}$ define certain constraints on the economic as well as diseaserelated parameters for maximization of the allied benefit due to administration of antiviral drug and vaccine. However, the rates of respective measures for maximum benefit can be 
found using model Equations (28) and (29) on substitution of optimal equilibrium $\left(S^{* *}, I^{* *}, R^{* *}\right)$, satisfying (33) and (34).

\section{Numerical simulation}

We make several interesting observations by numerically simulating the system Equations (1)-(3) for a range of parameter values. A major part of the simulation covers the effect of independent as well as integrated control of infection by application of antiviral drug and vaccine. The main parameter values listed in Table 1 are obtained from $[13,14,21,22,34]$ to reflect the dynamics of vertically transmitted disease, for example, HBV. We also consider $(0.99999,0.00001,0)$ as initial condition for simulation of the model.

With the above parameter values, the system asymptotically approaches towards the equilibrium $E_{1}^{*}=(0.3981,0.0160,0.5833)$, where the basic reproduction ratio $R_{0}=$ 2.5120 (Figure 2). As we have discussed and shown in Section 2, the equilibrium is endemic and stable when $R_{0}>1$.

We simulate the system at different values of rate of vaccine $u_{1}$. As it is shown in Figure 3, application of vaccination reduces the disease burden. In fact, the mean size of the infected population density is decreased with higher rate of vaccine. The transient behaviour of disease incidence also varies due to immunization. At a high rate of vaccination, the infected population density is reduced to a very low level initially and then it takes longer time to restore the steady-state value. In contrast to Figure 3, though the application of antiviral drug reduces the disease incidence, it does not alter the frequency and magnitude of initial oscillation in infected population density significantly (Figure 4). However, the antiviral drug works more slowly than vaccination.

The effect of two control measures on disease dynamics may be understood well if we consider Figure 5. It explains how control reproduction ratio $R_{\mathrm{c}}$ evolves with different rates of $u_{1}$ and $u_{2}$. It is seen that both vaccination and antiviral drug reduce the value of $R_{\mathrm{c}}$ effectively. But an integrated control works better than either of the control measures. We have also observed in Section 1 that the effect of integrated control actually is more than the sum of their independent effect. To explore the transient behaviour of disease dynamics on using integrated control, we plot the infected population density after 25 years of administration of vaccine and antiviral drug at different rates (Figure 6). In Figure 6, we see that initial oscillation in population dies out sooner if we use the antiviral drug at higher rate with application of vaccination. So, a mixed intervention strategy of vaccination and antiviral treatment is the best to reduce the disease burden more effectively.

Table 1. Parameter values used in numerical simulations.

\begin{tabular}{llll}
\hline Parameters & Description & Value & Reference \\
\hline$\mu$ & Birth (and death) rate per capita & 0.0121 & {$[34]$} \\
$k$ & Transmission rate & 10.22 & {$[13,34]$} \\
$q_{1}$ & Probability of infected newborns & 0.11 & {$[13]$} \\
$q_{2}$ & Probability of immune newborns & 0.1 & {$[14]$} \\
$\gamma$ & Recovery rate & 4.0566 & {$[13,34]$} \\
$\alpha$ & Rate of waning immunity & 0.1 & {$[14]$} \\
$m_{1}$ & Efficacy of vaccine & 0.81 & {$[24]$} \\
$m_{2}$ & Efficacy of antiviral drug & 0.71 & \\
$u_{1}$ & Rate of vaccination per year & $0-2$ & \\
$u_{2}$ & Rate of antiviral drug per year & $0-20$ & \\
\hline
\end{tabular}




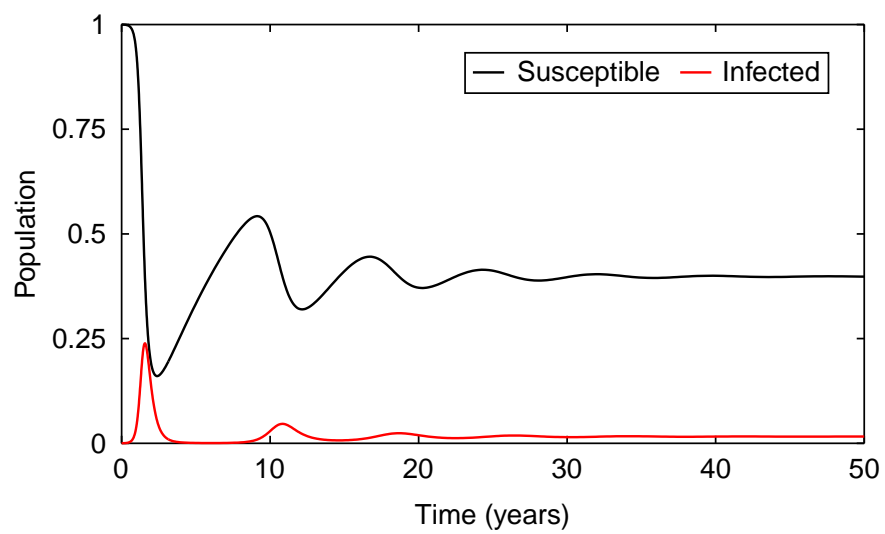

Figure 2. A representative time series of the susceptible and the infected, when no intervention strategies (vaccine or antiviral drug) is applied to the system. As discussed in Section 2, the system stabilizes to some endemic equilibrium $(0.3981,0.0160,0.5833)$. The basic reproduction ratio $R_{0}$ at this parameter value is 2.5120 .

We also experiment that how the disease evolves with change in rate of waning immunity $\alpha$. From Figure 7, it is observed that increase in $\alpha$ not only increases the mean size of disease incidence, but it also reduces the initial oscillation in population and more rapidly the population approaches towards the steady-state level. This is because high waning increases the probability of reinfection, and the individuals move from recovered compartment to susceptible compartment and this increases the disease prevalence by horizontal transmission.

To explore the dependence of probabilities $q_{1}$ and $q_{2}$ on disease dynamics, we plot the control reproduction ratio $R_{\mathrm{c}}$ with respect to $q_{1}$ and $q_{2}$ (Figure 8). It is seen that $R_{\mathrm{c}}$

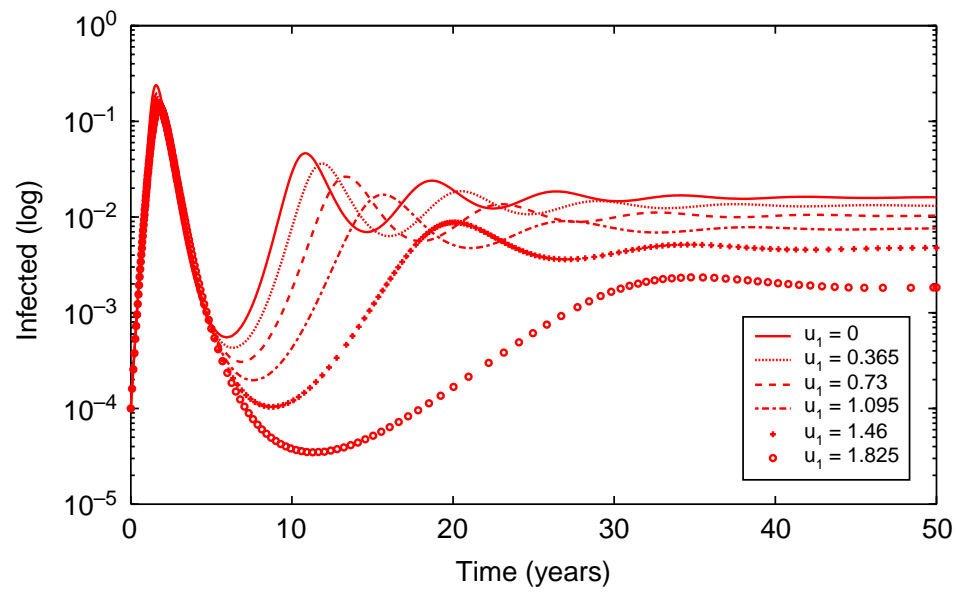

Figure 3. Infected population density at different rates of vaccination per day. Application of vaccination not only reduces the steady-state level of incidence, also decreases the mean size of infected population, and the disease restores more slowly once it is reduced to a very low level. 


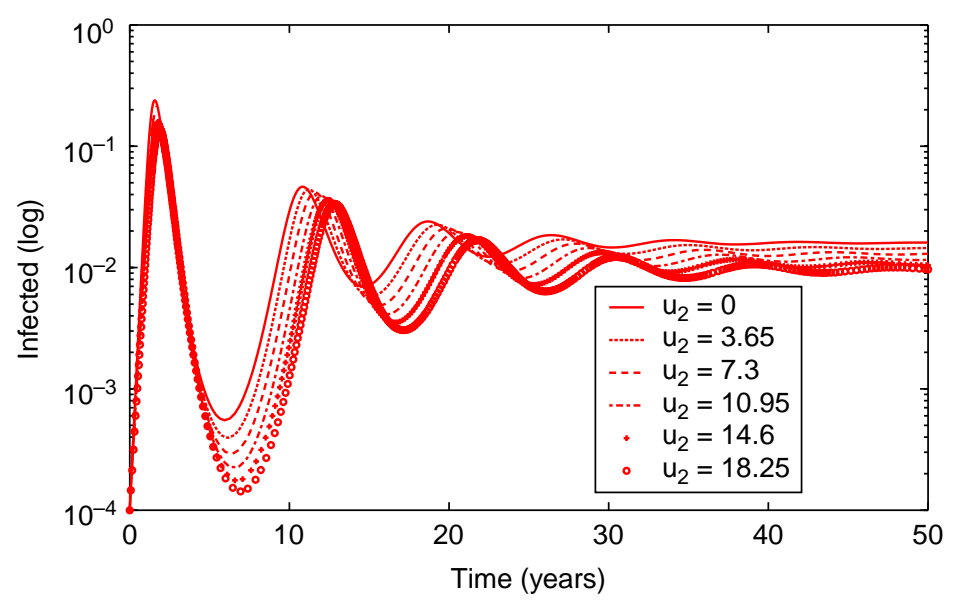

Figure 4. Infected population density at different rates of antiviral drug per day. In contrast to Figure 3, the steady-state level decreases more slowly with application of antiviral drug. Also, the frequency and magnitude of oscillation of disease incidence do not change with a high rate of antiviral drug.

increases as $q_{1}$ increases, but decreases as $q_{2}$ increases. Increase in $q_{1}$ increases the probability of infected newborns, and so $R_{\mathrm{c}}$ is increased. But, higher value of $q_{2}$ increases the proportion of immune newborns, which in turn reduces the per capita susceptible birth rate. This inhibits the horizontal transmission in unit time.

Hence, we observe that several disease-related parameters have nonlinear effect on the dynamics of disease. Especially, transmission rate, rate of waning immunity, proportion of perinatal infection, etc. have a major impact on the disease prevalence. Though we observe that the use of both vaccine and antiviral drug reduces the disease burden, the integrated control works more effectively than any other single control measure.

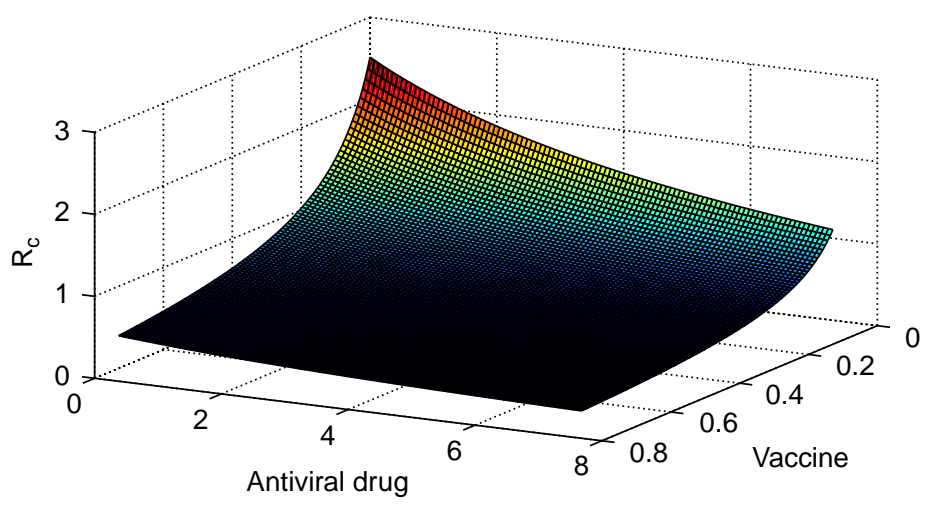

Figure 5. Control reproduction ratio $R_{\mathrm{c}}$ at different values of vaccination and antiviral drug. $R_{\mathrm{c}}=2.5120$, when there is no vaccination and antiviral drug (Figure 2). As it is also seen in earlier figures, application of vaccination reduces $R_{\mathrm{c}}$ more rapidly than antiviral drugs; though mixed intervention strategies always work better to reduce the disease burden. 


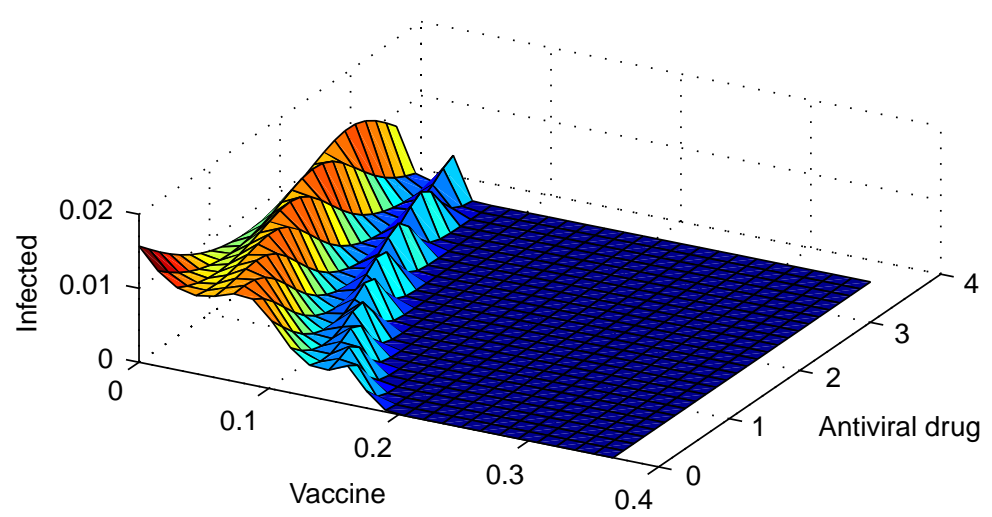

Figure 6. Infected population density after 25 years of application of vaccine and antiviral drug at different rates. It is also seen that application of antiviral drug with vaccine reduces the disease incidence in a very low level, and the higher the dose of drug, the sooner the incidence reduces to minimum level.

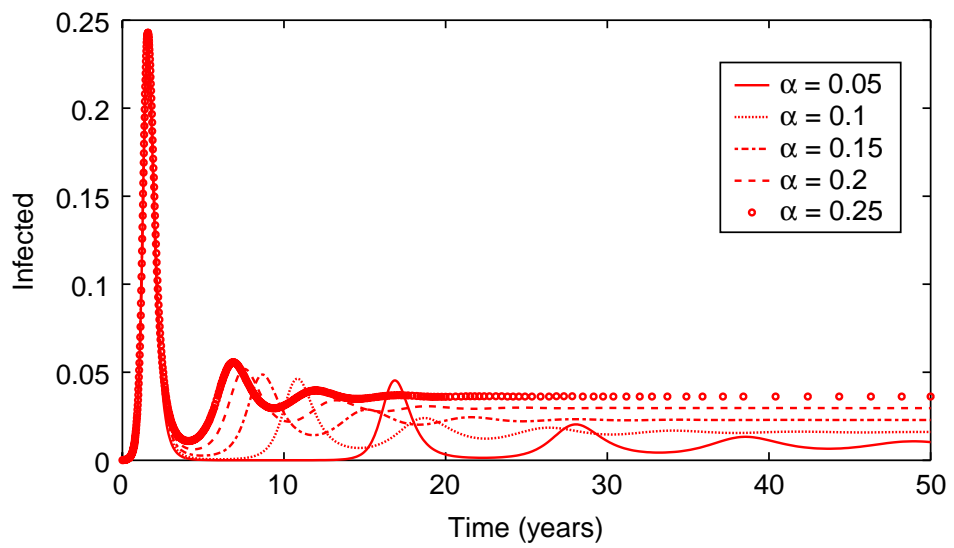

Figure 7. Infected population density at different values of waning immunity $\alpha$. High waning rate increases the infected population by increasing the susceptible pool. The mean size of infected population is increased at higher $\alpha$.

\section{Discussion}

In the present manuscript, we have proposed an SIR model of an infectious disease under two different control measures. The disease propagates from the infected to the susceptible in two different ways: through horizontal transmission or direct contact and through vertical transmission from mother to her offspring. There are infectious diseases, for instance hepatitis B and HIV, where there is a high probability in transmission of the disease from the infected mother to her child. Though there are many vaccines that reduce the disease transmission for most communicable diseases (presently there is no vaccine for HIV), several antiviral drugs are also available on the market which play a major role in the containment of the disease transmission.

We emphasize two key features in this paper and these are effective controls of disease transmission using integrated measures and optimal analysis of the cost-benefit model. 


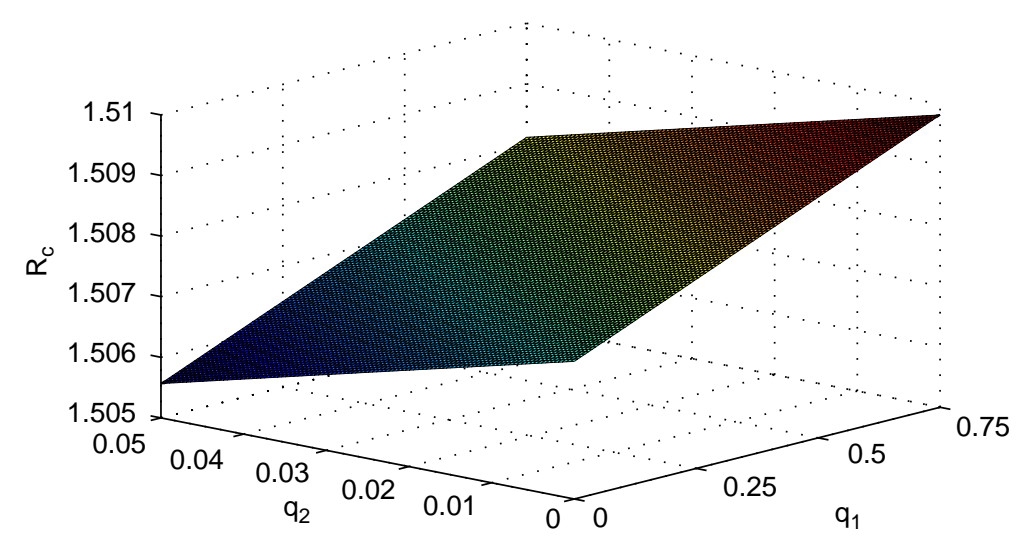

Figure 8. Control reproduction ratio $R_{\mathrm{c}}$ at different values of probabilities $q_{1}$ and $q_{2}$, whenever $u_{1}=0.0001$ and $u_{2}=0.005$. High $q_{1}$ increases the infected population density by increasing the proportion of infected newborns, whereas high $q_{2}$ decreases the infected population density by decreasing the proportion of susceptible newborns.

Although we use the simple SIR model to describe the disease dynamics, our studies demonstrate some interesting results that might have important clinical and epidemiological aspects.

Analysing as well as numerically simulating the model, we see that under certain parametric constraints, a combination of mixed control measures respond better rather than any other independent control. In a very recent paper by Zou et al. [34], it is reported that hepatitis B incidence in China is still increasing despite there being an effective vaccination programme since 1990 . HBV is a potentially life-threatening infectious disease in China these days. Though our model is simple to describe specific infections such as HBV, it shows that vaccination does not provide a permanent solution to control

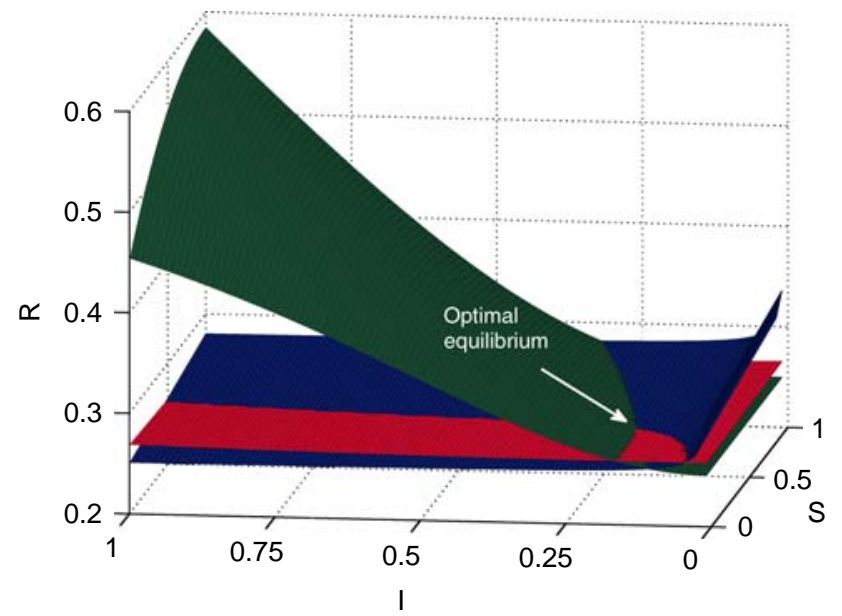

Figure 9. Figure exhibits optimal equilibrium at the intersection of three surfaces given by Equations (45)-(47), where $p=0.02, c_{\mathrm{M}}=0.03$ and $c_{\mathrm{v}}=0.015$ with other default parameters values given in Table 1. 
disease such as HBV, when there is a high probability of being re-infected. For example, some case studies have shown that there is a high chance of reinfection post liver transplantation in the case of HBV [29,30,33]. Also, vaccination of those at high risk has been of only limited success [33]. However, these two control measures have different mechanisms to control the overall transmission in population: vaccination reduces the transmission by reducing the susceptible population density, whereas antiviral drugs typically inactivate the enzymes needed for viral replication and reduce the rate of viral growth. This minimizes the severity of disease and reduces the chance of transmission to others. For instance, antiviral treatment cannot clear the infection, but they can stop the virus from replicating, and minimize liver damage such as cirrhosis and liver cancer in case of HBV [25].

Our result also shows that the use of antiviral drug with vaccination can reduce the initial oscillations in the disease prevalence. In fact, the oscillation dies out rapidly with higher rates of antiviral drug. We also show how the dynamics of disease evolves depending on probability $\left(q_{1}\right)$ of infected newborns and probability $\left(q_{2}\right)$ of immune newborns. In fact, the control remains more effective with higher values of $q_{2}$, whereas it works poorly with higher values of $q_{1}$. Thus, if it is possible to make a more efficacious vaccine to provide immunity that inherits from mother to child, then it might be helpful in controlling the overall transmission of the disease.

Our work has some considerable scopes to extend further. For instance, it may be considered as a more explicit model of vertical transmission with age-structured framework or for emphasizing any specific disease. This might be helpful to quantify particular parameter(s) that regulate the transmission or are most responsible for an effective control measure. Also, disease with vertical transmission potentially depends on the birth in population. We have assumed stable population, but it might be interesting to look at how the disease propagates in a population with a fluctuating birth rate. It is also of interest to consider periodic contact rates in disease transmission and to design the immunization programme accordingly. Another important aspect of infection-prevalence dynamics is the role of case importation. This is the only source of disease burden once local chains of transmission have been interrupted through herd immunity due to vaccination. Therefore, inclusion of case importation should potentially modify the disease dynamics once herd immunity develops through the immunization programme.

However, modelling the disease and quantifying the rates of control measures do not immediately assess the optimal control of the disease in the population. The essence of management of disease in a population is to make the optimal decision subject to the realistic constraints, so that it reduces the health care costs and/or improves quality-of-life for individuals by preventing or minimizing the effects of a disease through integrative care. This, in fact, is none other than an optimization problem. However, in practice there are enormous difficulties in quantifying the variables, the objectives and the constraints in a given decision problem. Here, we have focused our attention on the task of formulating an optimal policy when a decision problem has already been defined in a mathematical form.

In this regard, we may mention that the numerical solution of the optimal control problem is much harder than that for the standard optimal control problem. In this case, the steady-state optimization plays an important role. It has added advantage of being easily implemented to the real-world problem. To keep the state of the system at such an optimal steady state, if possible one can employ a globally stable control policy whenever the state is displaced from the optimal steady state. Another important theoretical development in the late 1960s was the derivation of necessary conditions for singular control. Singular 
control occurs in an optimal control problem in which one or more of the control variables appear linearly in system dynamics and the objective function. In biological problems, the concept of singular control is important because control variables often occur linearly in the system dynamics. References in this connection may be given to the work of [11].

In this paper, we perform the optimal analysis of a cost-benefit model with two control parameters and find out the necessary conditions on the parameters to make the control optimum. For the same set of default parameter values, we show in Figure 9 that surfaces defined by solutions of the Equations (45)-(47) intersect at a single point, which denotes optimal values of susceptible, infected and recovered. We can, however, generalize the same approach of optimization in the application to models with more than two control parameters.

\section{Acknowledgements}

We would like to thank Dr Chris Bauch, University of Guelph, for helpful discussions on the manuscript. We are also grateful to two anonymous reviewers for their valuable comments and suggestions to improve the manuscript.

\section{References}

[1] N. Bailey, The Mathematical Theory of Infectious Diseases, 2nd ed., Hafner, New York, 1975.

[2] F. Baryarama, J.Y.T. Mugisha, and L.S. Lubooobi, Mathematical model for HIV/AIDS with complacency in a population with declining prevalence, Comput. Math. Methods Med. 7 (2006), pp. 27-35.

[3] P.E. Beitune, G. Duarte, S.M. Quintana, E.A. Figueiró-Filho, A.C. Marcolin and R. Abduch, Antiretroviral therapy during pregnancy and early neonatal life: Consequences for HIVexposed, uninfected children, Braz. J. Infect. Dis. 8 (2004), pp. 140-150.

[4] S. Bhattacharyya and D.K. Bhattacharya, A more realistic approach to pest management, Bull. Math. Biol. 69 (2007a), pp. 1277-1310.

[5] S. Bhattacharyya and D.K. Bhattacharya, An improved integrated pest management model under 2-control parameters (sterile male and pesticide), Math. Biosci. 209 (2007b), pp. 256-281.

[6] B.S. Bloom, A.L. Hillman, A.M. Fendrick, and J.S. Schwartz, A reappraisal of hepatitis B virus vaccination strategies using cost-effectiveness analysis, Ann. Intern. Med. 118 (1993), pp. 298-306.

[7] F. Brauer, Models for diseases with vertical transmission and nonlinear population dynamics, Math. Biosci. 128 (1995), pp. 13-24.

[8] S.N. Busenberg and K.L. Cooke, Models of Vertically Transmitted Diseases with Sequencialcontinuous Dynamics, in Nonlinear Phenomena In Mathematical Sciences, V. Lakshmikantam, ed., Academic Press, New York, 1982, pp. 179-187.

[9] S.N. Busenberg, K.L. Cooke, and M. Pozio, Analysis of a model of a vertically transmitted disease, J. Math. Biol. 17 (1983), pp. 305-329.

[10] L.L. Cavalli-sforza and M.W. Feldman, Cultural transmission and evolution: A quantitative approach, Monographs in Population Biology Princeton, Univ. Press 16 (1983).

[11] C.W. Clark, Economically optimal policies for the utilization of biologically renewable resources, Math. Biosci. 12 (1971), pp. 245-260.

[12] A. d'Onofrio, Globally stable vaccine-induced eradication of horizontally and vertically transmitted infectious diseases with periodic contact rates and disease-dependent demographic factors in the population, Appl. Math. Comput. 140 (2003), pp. 537-547.

[13] W.J. Edmunds, G.F. Medley, and D.J. Nokes, The transmission dynamics and control of hepatitis B virus in the Gambia, Stat. Med. 15 (1996a), pp. 2215-2233.

[14] W.J. Edmunds, G.F. Medley, and D.J. Nokes, Vaccination against hepatitis B virus in highly endemic area: Waning vaccine-induced immunity and the need for booster doses, Trans. R. Soc. Trop. Med. Hyg. 90 (1996b), pp. 436-440. 
[15] M. El Doma, Stability analysis of a general age-dependent vaccination model for a vertically transmitted disease under the proportionate mixing assumption, IMA J. Math. Appl. Med. Biol. 17 (2000), pp. 119-136.

[16] G. Fattovich, F. Bortolotti, and F. Donato, Natural history of chronic hepatitis B: Special emphasis on disease progression and prognostic factors, J. Hepatol. 48 (2008), pp. 335-352.

[17] S. Ghosh and D.K. Bhattacharyya, Optimization in microbial pest control: An Integrated approach, Appl. Math. Model. 34 (2010) 1382-1395.

[18] B.S. Goh, J. Leitman, and T.L. Vincent, Optimal control of prey-predator systems, Math. BioSci. 19 (1974), pp. 263-286.

[19] B. Hethcote, H. Stech, and P. van den Driessche, Periodicity and Stability in Epidemic Models, in Differential Equations and Applications to Ecology, Epidemics, and Population Problems, S.N. Busenberg and K.L. Cooke, eds., Academic press, New York, 1981, pp. 65-82.

[20] E.O. Jonesa, A. Whiteb, and M. Boots, Interference and the persistence of vertically transmitted parasites, J. Theor. Biol. 246 (2007), pp. 10-17.

[21] E.B. Keeffe, Acute hepatitis A and B in patients with chronic liver disease: Prevention through vaccination, Am. J. Med. 118 (2005), pp. 21S-27S.

[22] K. Krogsgaard et al., Relation between treatment efficacy and cumulative dose of alpha interferon in chronic hepatitis B, J. Hepatol. 25 (1996), pp. 795-802.

[23] M.Y. Li, H.L. Smith, and L. Wang, Global dynamics of an SEIR epidemic model with vertical transmission, SIAM J. Appl. Math. 62 (2001), pp. 58-69.

[24] MMWR, Progress in hepatitisB prevention through universal infant vaccination in China 1997-2006, Morb. Mortal. Wkly. Rep. 56(18) (2007), pp. 441-445.

[25] R. Naresh, A. Tripathi, and S. Omar, Modelling the spread of AIDS epidemic with vertical transmission, Appl. Math. Comput. 178 (2006), pp. 262-272.

[26] B.J. Nassal, Hepatitis B virus replication, World J. Gastroenterol. 13(1) (2007), pp. 48-64.

[27] R.P. Perrillo, Chronic hepatitis B: A critical appraisal of current to therapy, Clin. Gastroentrol. Hepatol. 4 (2006), pp. 233-248.

[28] M.R. Razvan, The dynamics of a vertically transmitted disease, J. Prime Res. Math. 3 (2007), pp. 201-209.

[29] J. Rosenau et al., Lamivudine and low-dose hepatitis B immune globulin for prophylaxis of hepatitis $B$ reinfection after liver transplantation possible role of mutations in the YMDD motif prior to transplantation as a risk factor for reinfection, J. Hepatol. 34 (2001a), pp. 895-902.

[30] J. Rosenau et al., Successful hepatitis B reinfection prophylaxis with Lamivudine and hepatitis $b$ immune globulin in patients with positive $H B V-D N A$ at time of liver transplantation, Transplant. Proc. 33 (2001b), pp. 3637-3638.

[31] H.R. Thieme, Mathematics in Population Biology, Princeton University Press, Princeton, NJ, 2003.

[32] D.L. Veenstra et al., Evaluating anti-viral drug selection and treatment duration in HBeAgnegative chronic hepatitis B: A cost-effectiveness analysis, Aliment. Pharmacol. Ther. 27 (2008), pp. 1240-1252.

[33] T.L. Wright and J.Y.N. Lau, Clinical aspects of hepatitis B virus infection, Lancet 342 (1993), pp. $1340-1344$.

[34] L. Zou, W. Zhang, and S. Ruan, Modelling the transmission dynamics and control of hepatitis B virus in China, J. Theor. Biol. 262 (2010), pp. 330-338. 


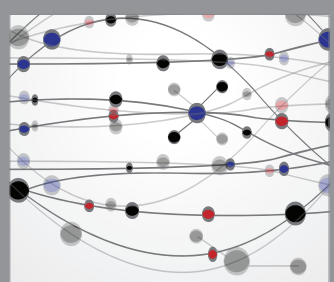

The Scientific World Journal
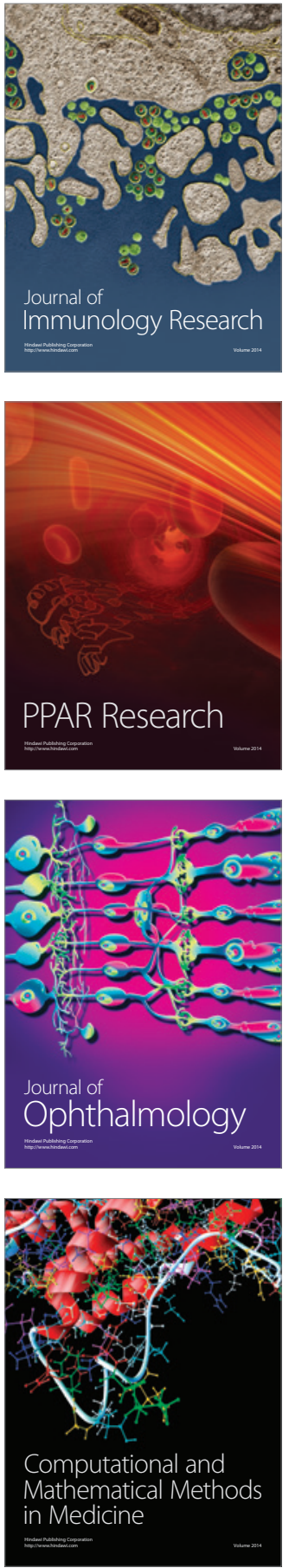

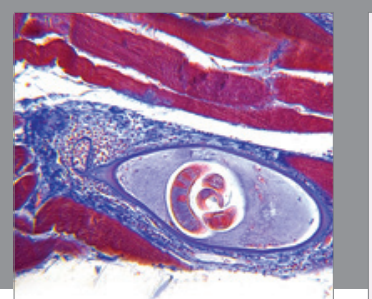

Gastroenterology Research and Practice

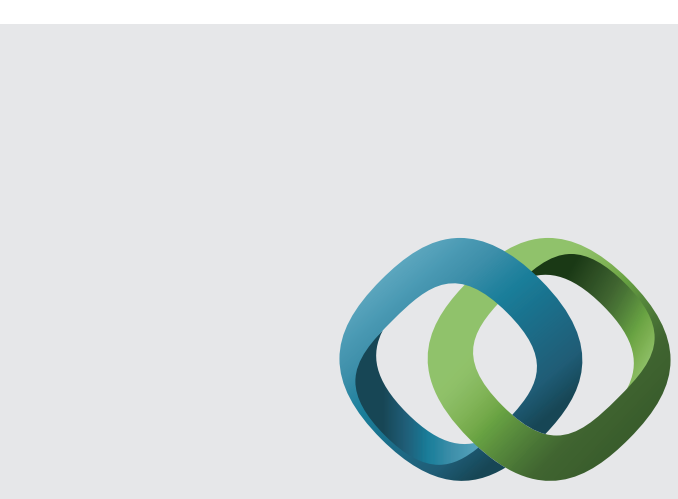

\section{Hindawi}

Submit your manuscripts at

http://www.hindawi.com
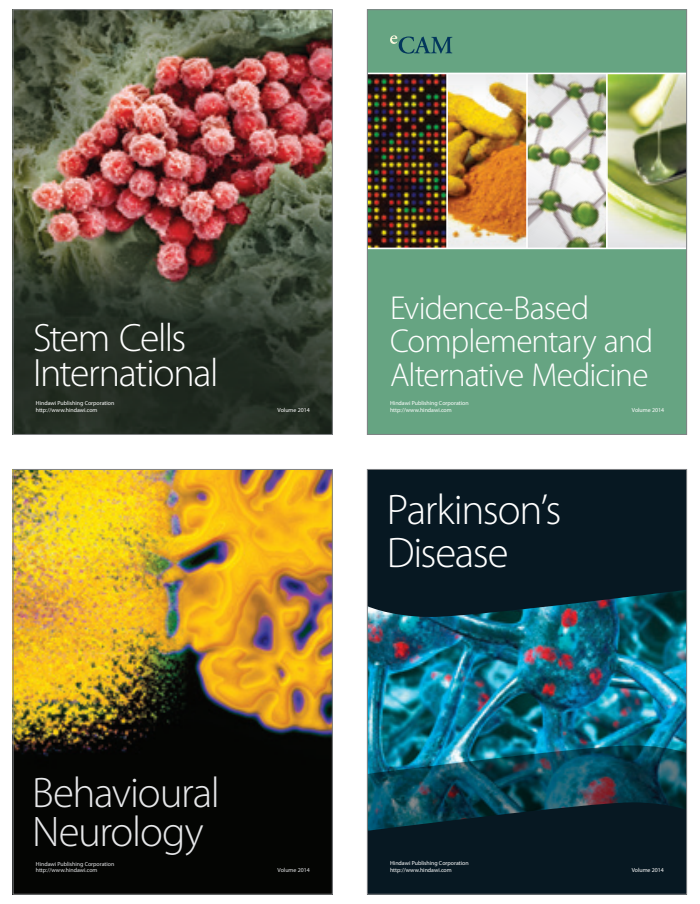
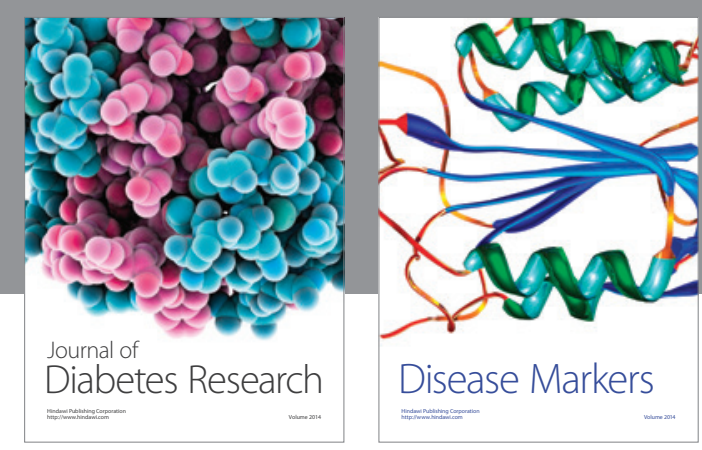

Disease Markers
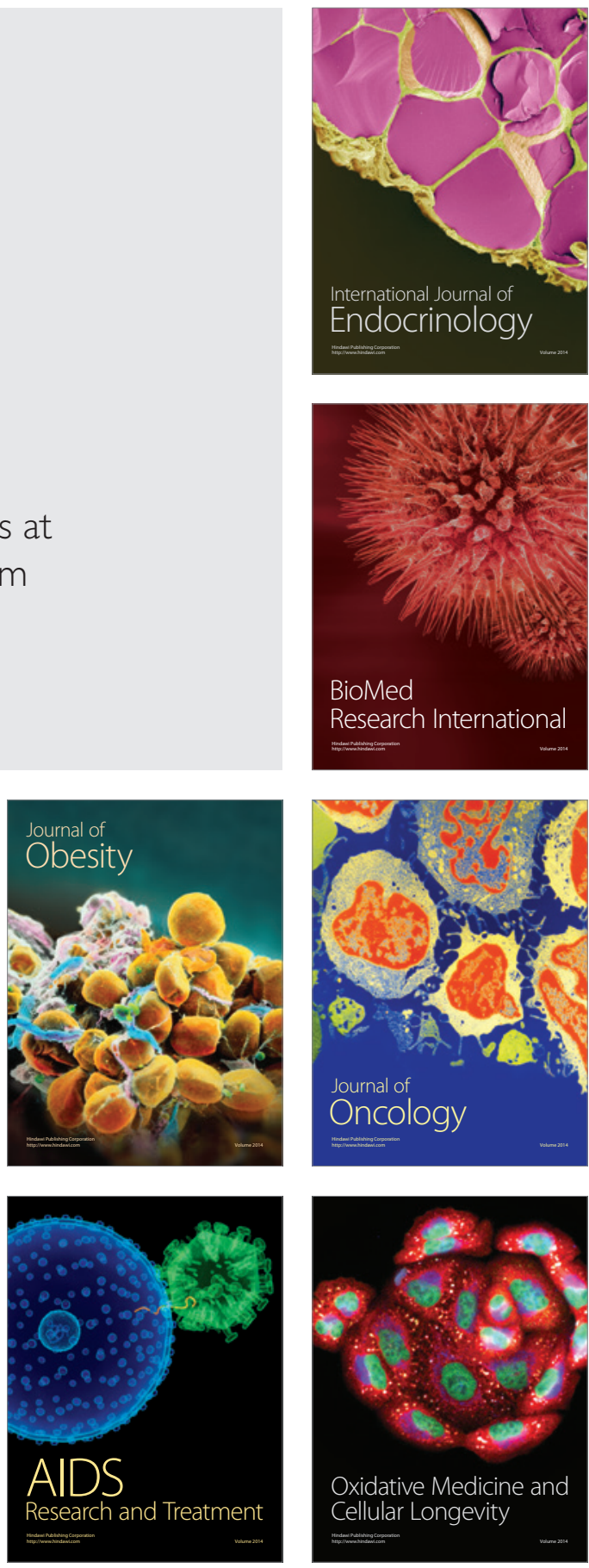